\title{
Geriatric oncology: where are we now?
}

\section{Laura McGuinness, Commissioning Editor, speaks to Silvio Monfardini}

Professor Silvio Monfardini is currently the Chief of the Geriatric Oncology Program at the Istituto Palazzolo (Milan, Italy). Professor Silvio Monfardini is the author of over 500 publications, over 300 of which have been published in indexed journals. In the field of geriatric oncology he has produced 55 indexed publications. He is also the author of the 'Manual of Medical Oncology (International Union Against Cancer)' and has contributed to other oncology manuals and text books. Throughout his career Silvio Monfardini has worked in the fields of non-Hodgkin's lymphomas, Hodgkin's disease, chronic myeloid leukemia, solid tumors (in particular testicular carcinomas) Phase I-II studies and tumors in the elderly. In the year 2003-2004 he served as the President of the International Society of Geriatric Oncology (SIOG). In 2006, he received the Paul Calabresi award from the International Society of Geriatric Oncology. He is also a valuable member of the Aging Health editorial board.

\section{- A large proportion of cancer patients are over the age of 65 years, do cancers present differently in the older age group?}

The biological characteristics of cancers in older patients are generally very similar to those of cancers present in younger adults, with the possible exception of breast cancer. In breast cancer, the percentage of positive endocrine receptors is higher in older women, which consequently means that the disease can be more successfully treated with hormone therapy.

In general, the differences between the two patient groups are not really due to the morphology of the cancer, but have more to do with the tolerability of the patient to different treatment options.

There is often a misconception that tumors progress more slowly in the elderly than in younger adults. This is often used as a reason not to treat them, but this is not true at all. Tumors in the elderly have the same aggressiveness and speed of growth as in younger adults. It is a false belief that they are different.

\section{- How does cancer management vary between older patients and younger patients?}

Age-associated conditions, such as comorbidity or a decrease of functional status, can lead to limitations in medical treatment administration in older patients.

You cannot always apply the same therapy to older patients as you would to all adult patients, but you should be able to overcome this with alternative solutions.
A full geriatric evaluation should be carried out to decide whether you should treat, the aggressiveness of the treatment or whether to simply give palliative therapy. This preliminary work requires patience, more time and ultimately more economic support than treating a younger patient. A full assessment will require at least $45 \mathrm{~min}$; when you imagine a busy oncology outpatient department, where there are many patients waiting for chemotherapy, you can see that dedicating this time to the elderly patient can become difficult.

This is the main reason why managing cancer in an older patient is different to managing cancer in a younger patient; but we should always give the patient as much treatment as they can tolerate.

\section{- Is an elderly cancer patient as likely to be put forward for surgery as a younger patient? Should this be the case?}

It depends on the surgeon. Some surgeons have more experience operating on older patients; they frequently work together with geriatricians and cooperate with anesthesiologists who are used to dealing with very old patients. Other surgeons are not so willing or able to operate on elderly patients. As well as varying between surgeons, there is also great variability between different countries.

However, the progressive aging of the population will inevitably lead to the increased practice of geriatric surgery. In Italy, many universities teach medical students about geriatric surgery and this has led to more widespread acknowledgement that surgery in the elderly can be a viable option. This area is progressing at the moment, but although the situation is improving there is still more that can be done.
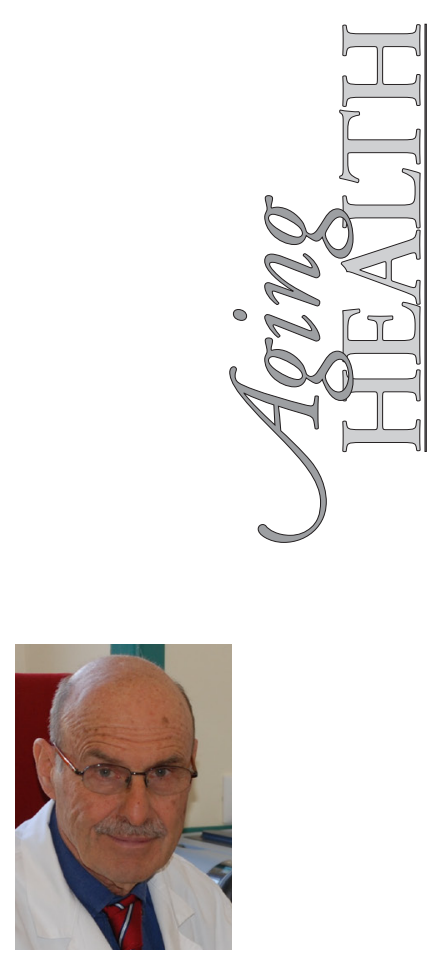

Silvio Monfardini Geriatric Oncology Program Istituto Palazzolo, Milan, Italy smonfardini@dongnocchi.it 


\section{How does prescribing anticancer drugs vary between older and younger patients?}

In older patients some drugs with less toxicity can be used successfully, these preferred drugs are known as 'elderly friendly'. We do use other drugs, but we have to be more careful. In some solid tumors single sequential agents can be used instead of combination chemotherapy to reduce the toxic signs. The use of initially reduced doses to test tolerance and then gradually increasing dosage is also a common practice.

\section{How does palliative care for the older adult differ from palliative care for a younger patient?}

Ideally there should be no difference concerning palliative care; however, too often pain in the elderly is not adequately treated, especially in nursing homes. From my experience, this could be attributed to the older patients themselves not being willing to complain or not wishing to become a burden and so simply putting up with the pain, not realizing that there are painkillers that could help them. Some pain therapy should be taken preventatively, but older patients are less inclined to self-medicate unless they are already experiencing pain, this may then be a little late as pain control takes time to work.

\section{- Should all elderly people be treated in the same way?}

This is certainly not the case; each person should be treated as an individual. Not all elderly people should be treated with the same intensity. When deciding how to treat a patient it is important that their functional age be considered as opposed to relying solely on their chronological age. The clinician must make a multidimensional geriatric evaluation; this allows them to distinguish fit, vulnerable and frail patients. In the field of medical oncology, older patients who are fit can receive chemotherapy in its full dose, equal to the treatment given to younger adult patients. Vulnerable patients can be treated with an adapted chemotherapy or initially reduced doses. Some frail patients can be treated with chemotherapy or hormonal therapy or with simple palliation. In all cases, geriatric evaluation is required to determine the appropriate treatment approach.
Why are elderly patients less likely to be put forward for clinical trials?

To carry out clinical trials in the elderly requires special protocols conceived specifically for older patients. Special support in the transportation of patients and dedicated relatives are also often necessary.

It is also more difficult to sign older people up for clinical trials because it can be more difficult to obtain informed consent, owing to the decreased mental status and reduced understanding of some patients.

In conclusion, it if often more time consuming and expensive to include elderly patients in trials, and frequently trial budgets do not allow for this.

\section{- Can we really extrapolate oncology trial} results to the elderly population?

Oncology trials cannot be directly extrapolated to the elderly population since they are conducted in a selected population. Even if older patients are included, it is usually only fit older patients who are receiving the planned treatment. These fit older patients are selected for a low comorbidity score and good performance status, and therefore react to the treatment in a very similar way to younger adults.

Sometimes studies will claim that older patients have been included, but it is a big mistake to say that the results apply to the older population if only fit older people have been studied.

\section{a Going forward, how do you envisage that we solve this problem?}

We need studies to evaluate a population of patients from the real world, not selected patients. The solution may be that we need to conduct observational trials in an unselected population of patients, who are then homogeneously treated.

\section{- Do you think that geriatric oncology is finally being recognized as a field in its own right?}

At the moment I think it is still too early to think that there is widespread recognition of geriatric oncology. Our best allies in pushing the importance of the field forward are the geriatricians, but in many countries they do not exist. The situation is more favorable in countries where the practice of geriatrics has recognition.

The field of Geriatric Oncology is gaining more recognition: for example Dr Matti Aapro, 
Executive Director of the International Society for Geriatric Oncology (SIOG), will be given the BJ Kennedy award at the American Society of Clinical Oncology meeting this year. This award is given for providing outstanding leadership of major importance to the field of geriatric oncology.

- How are we encouraging young doctors to train in the field of geriatric oncology?

There are already many young medical oncologists very attentive to the care, clinical problems and research concerning older cancer patients.

The best approach to nurturing interest in young doctors is often trying to discuss the treatment of older patients with cancer with young fellows, trying to encourage them to see the patient as a whole person and take into consideration all of their problems when developing a personalized approach.

The SIOG also has a lot of initiatives and has been organizing training courses around Europe and also in East Asia and in Australia.

\section{- Can you tell me more about the work you are carrying out at the moment?}

So far geriatric information has been brought into the oncological setting around the world to treat older patients with cancer, for example through the use of multidimensional geriatric evaluation before the treatment choice. But it is also possible for a trained medical oncologist to administer cancer chemotherapy in a geriatic institute, bringing oncological knowledge into geriatrics. At present, we are carrying this out at the Istituto Palazzolo (Milan, Italy), which is a geriatric and rehabilitation institute that is trying to introduce the medical treatment of cancer patients into the field of geriatrics. This has been carried out in the USA and Japan, but our institution is one of the first to do this in Italy. The main difficulty lies in the fact that the culture, mentality and organization modalities are so different in the two disciplines.

\section{What are your hopes for the field over the coming years?}

My hope is that this experiment, introducing oncology to the geriatric department, will be useful and serve to open a new methodological approach to the problem. I am currently involved with an international task force, set up by SIOG, to study "the organization of clinical activity of geriatric oncology". In general, I hope that a better and more uniform approach to the organization of geriatric oncology might be set up; but this will only be possible when specific funds are available. This has been made possible in some countries such as France and the USA; I consider these to be the success stories. I hope to encourage political decisions to implement better organization in geriatric oncology, and that these decisions will be made, in all countries in Europe at least.

\section{Financial \& competing interests disclosure}

The author has no relevant affiliations or financial involvement with any organization or entity with a financial interest in or financial conflict with the subject matter or materials discussed in the manuscript. This includes employment, consultancies, honoraria, stock ownership or options, expert testimony, grants or patents received or pending, or royalties.

No writing assistance was utilized in the production of this manuscript. 